\title{
ENERGIE ET ENVIRONNEMENT
}

\author{
Axel Gautier \\ Département d'économie \\ HEC-Ecole de gestion de l'ULg
}




\section{Plan de l'exposé}

- Les objectifs: la stratégie Europe 2020

- Le contexte: le marché libéralisé de l'électricité

- Les défis: intégrer les énergies renouvelables

- Conclusions 


\section{LA STRATÉGIE EUROPE 2020}




\section{Les objectifs énergétiques de la stratégie Europe 2020}

- Réduction des émissions de gaz à effet de serre de $\mathbf{2 0} \%$ (voire de $\mathbf{3 0} \%$, si les conditions le permettent) par rapport à 1990

- Utilisation d'énergie provenant de sources renouvelables à hauteur de $20 \%$

- Augmentation de $\mathbf{2 0} \%$ de l'efficacité énergétique 


\section{Greenhouse gas emissions}

index base year $=100$

Total Greenhouse Gas Emissions (in CO2 equivalent) indexed to 1990

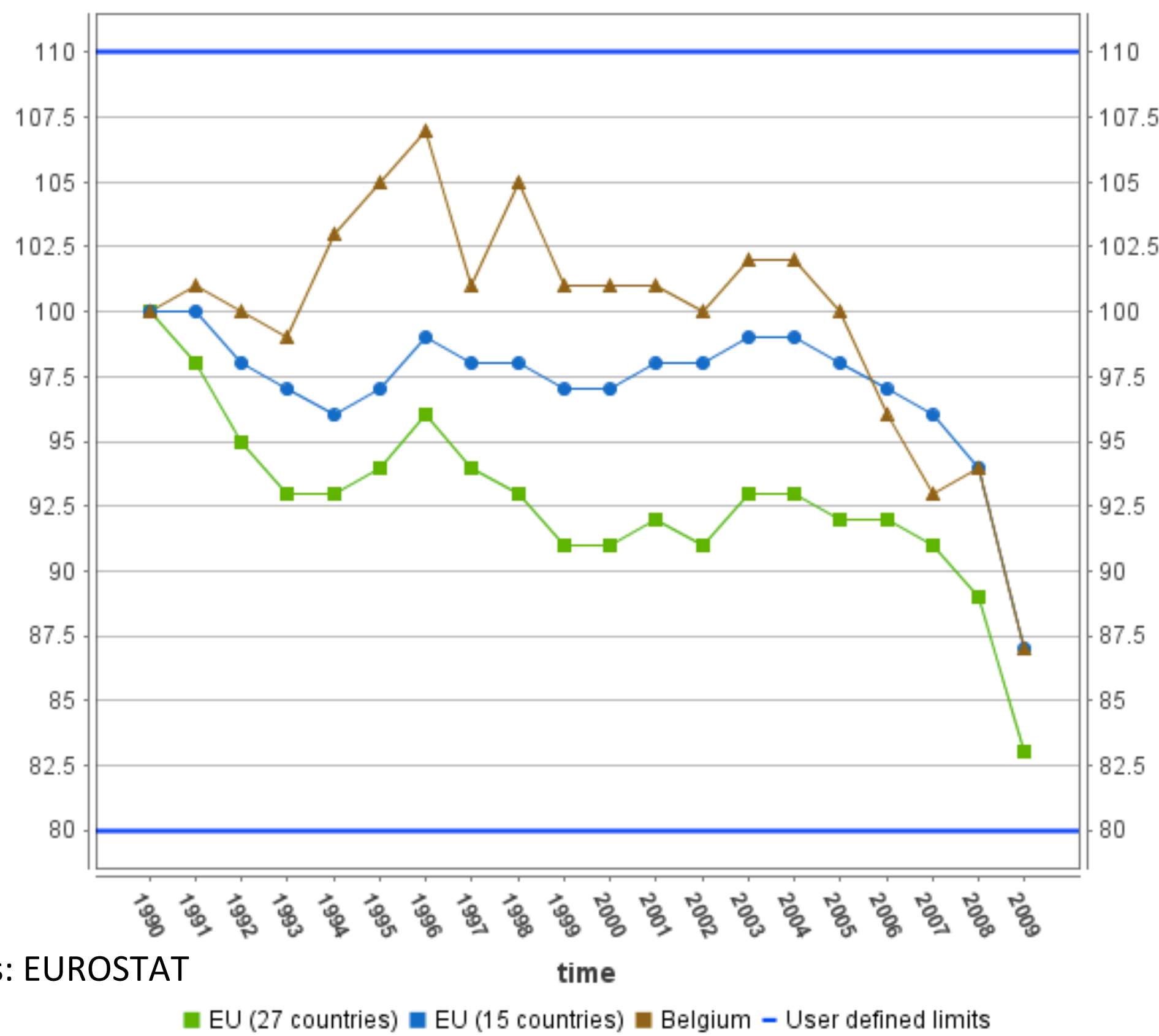

Données: EUROSTAT

- EU (27 countries) $\square$ EU (15 countries) - Belgium - User defined limits 


\section{\% énergie renouvelable, Belgique}

Share of renewable energy in gross final energy consumption $\%$

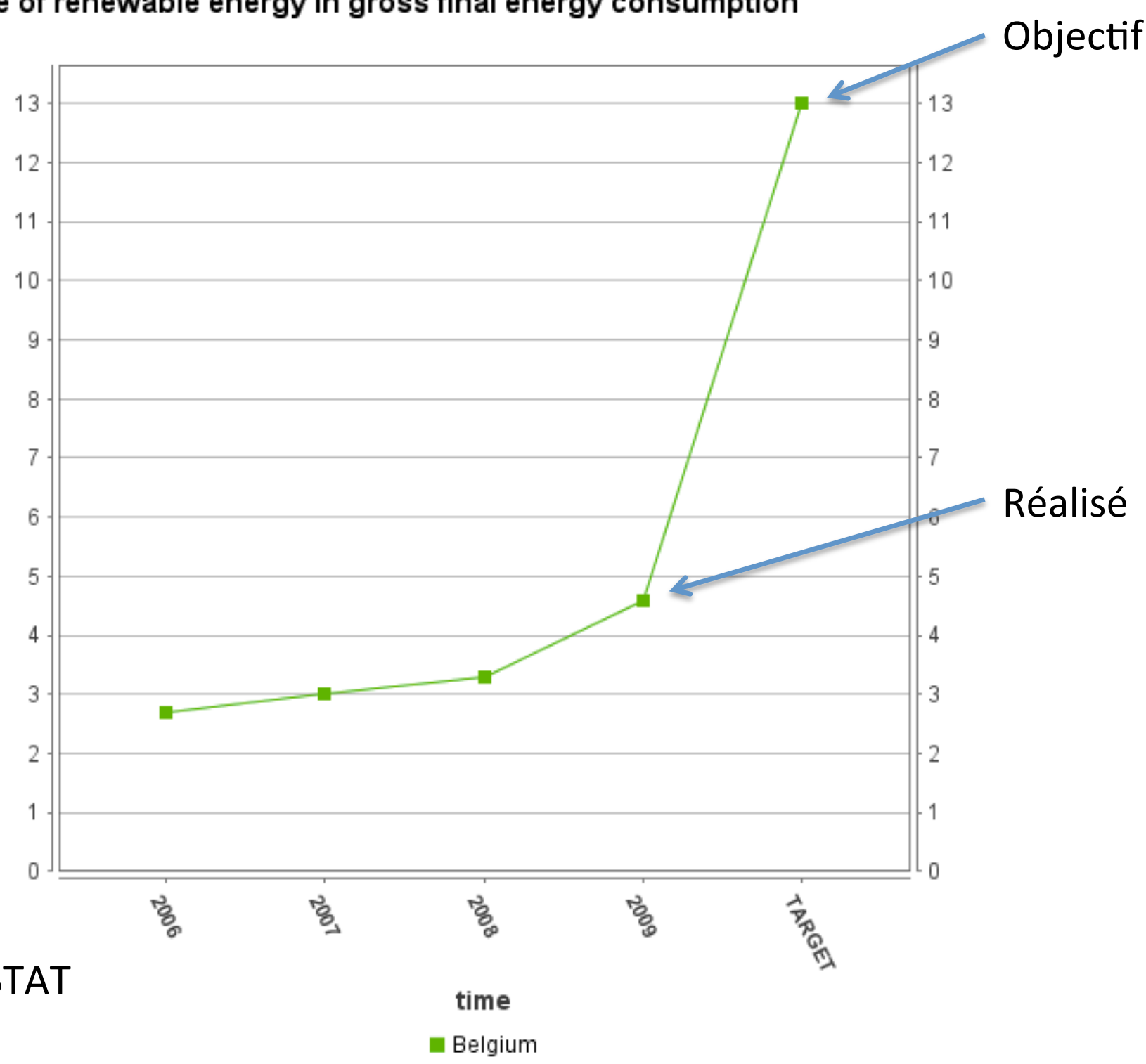

Données: EUROSTAT 


\section{$\%$ énergie renouvelable}

ihare of renewable energy in gross final energy consumption

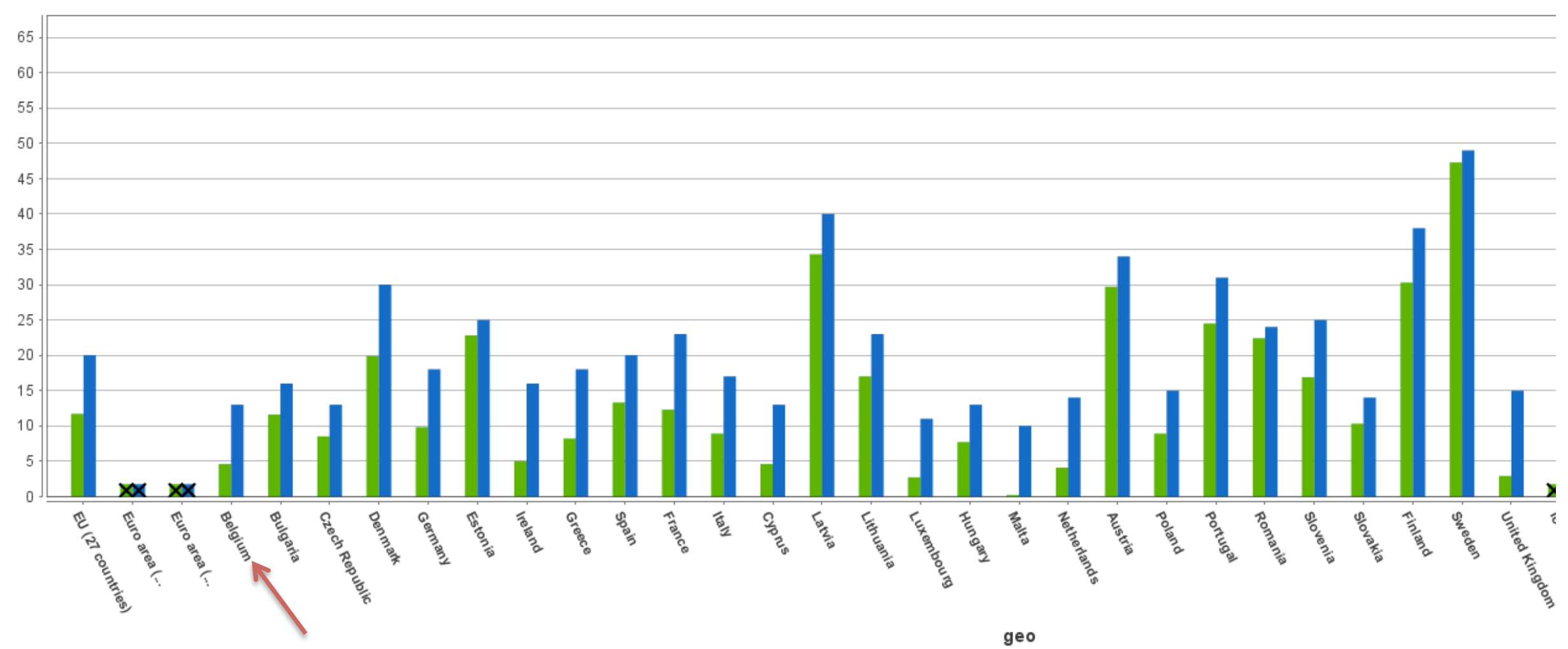

॥ 2009 = TARGET $\mathbf{X}$ No data

Données: EUROSTAT 


\section{Electricity generated from renewable sources}

$\%$ of gross electricity consumption

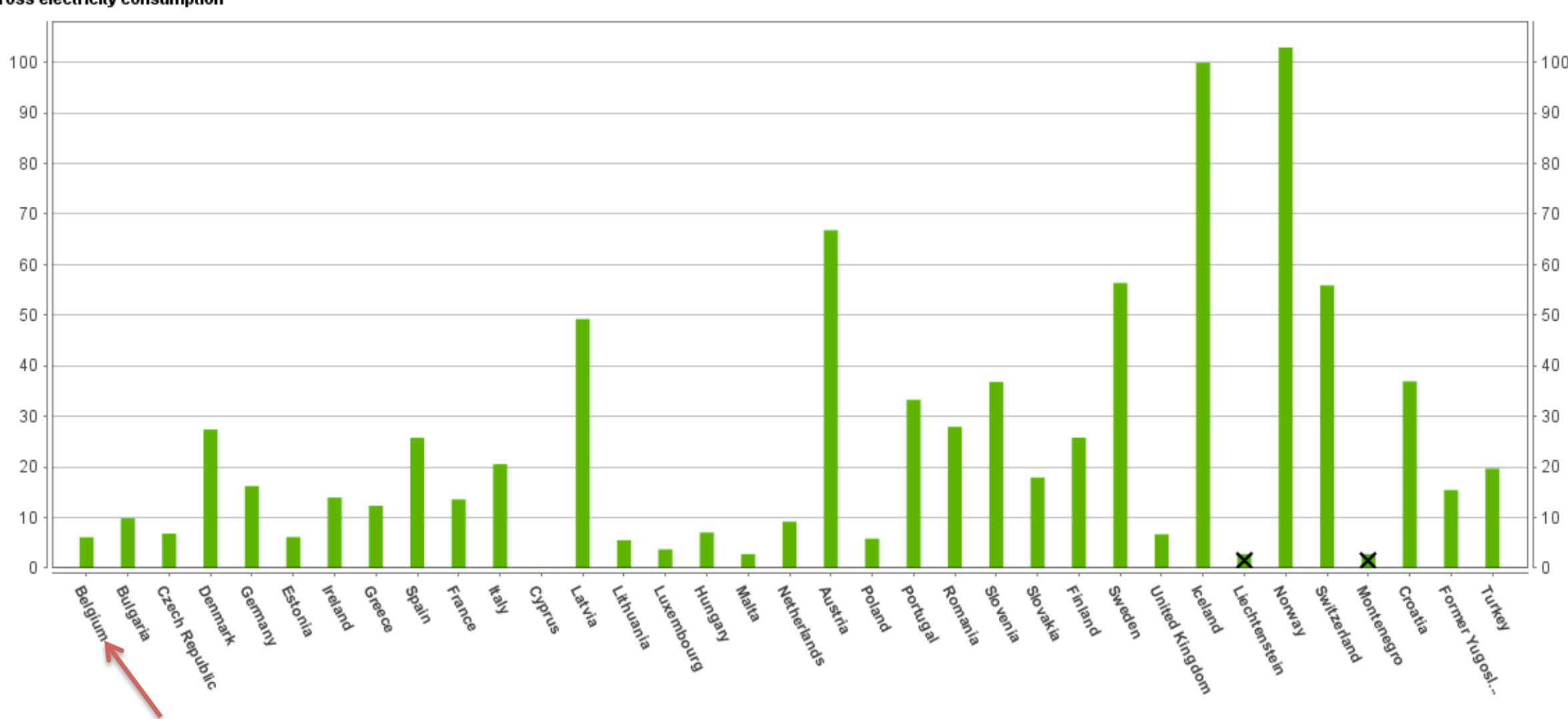

geo

Exceptions: IS (1997) 


\section{Electricity generated from renewable sources}

\section{$\%$ of gross electricity consumption}

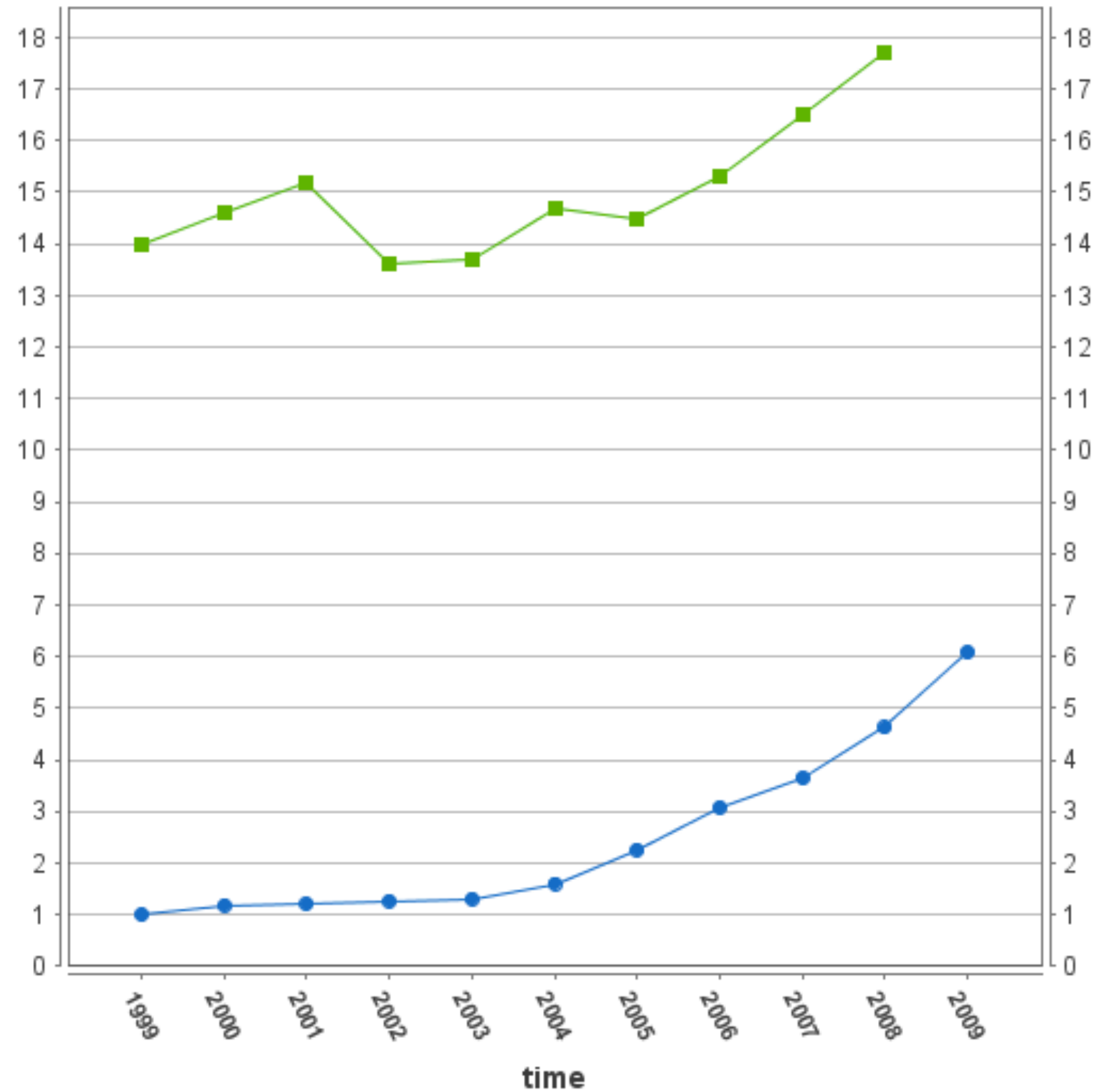

EU (15 countries) $\square$ Belgium * Unavailable data is ignored 


\section{Energy intensity of the economy}

Gross inland consumption of energy divided by GDP (kilogram of oil equivalent per 1000 Euro)

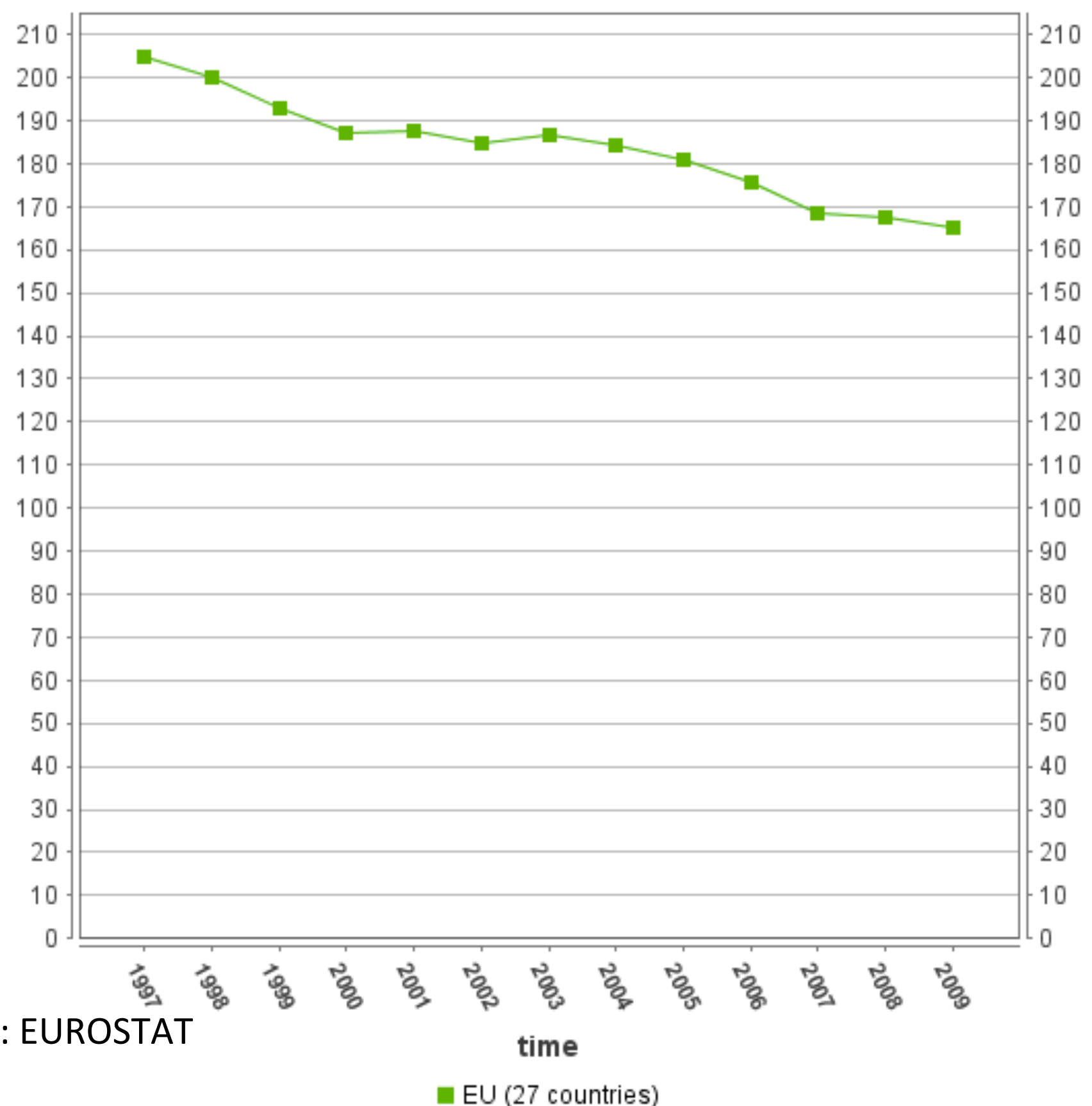

Données: EUROSTAT

EU (27 countries) 


\section{Electricity consumption of households}

1000 toe

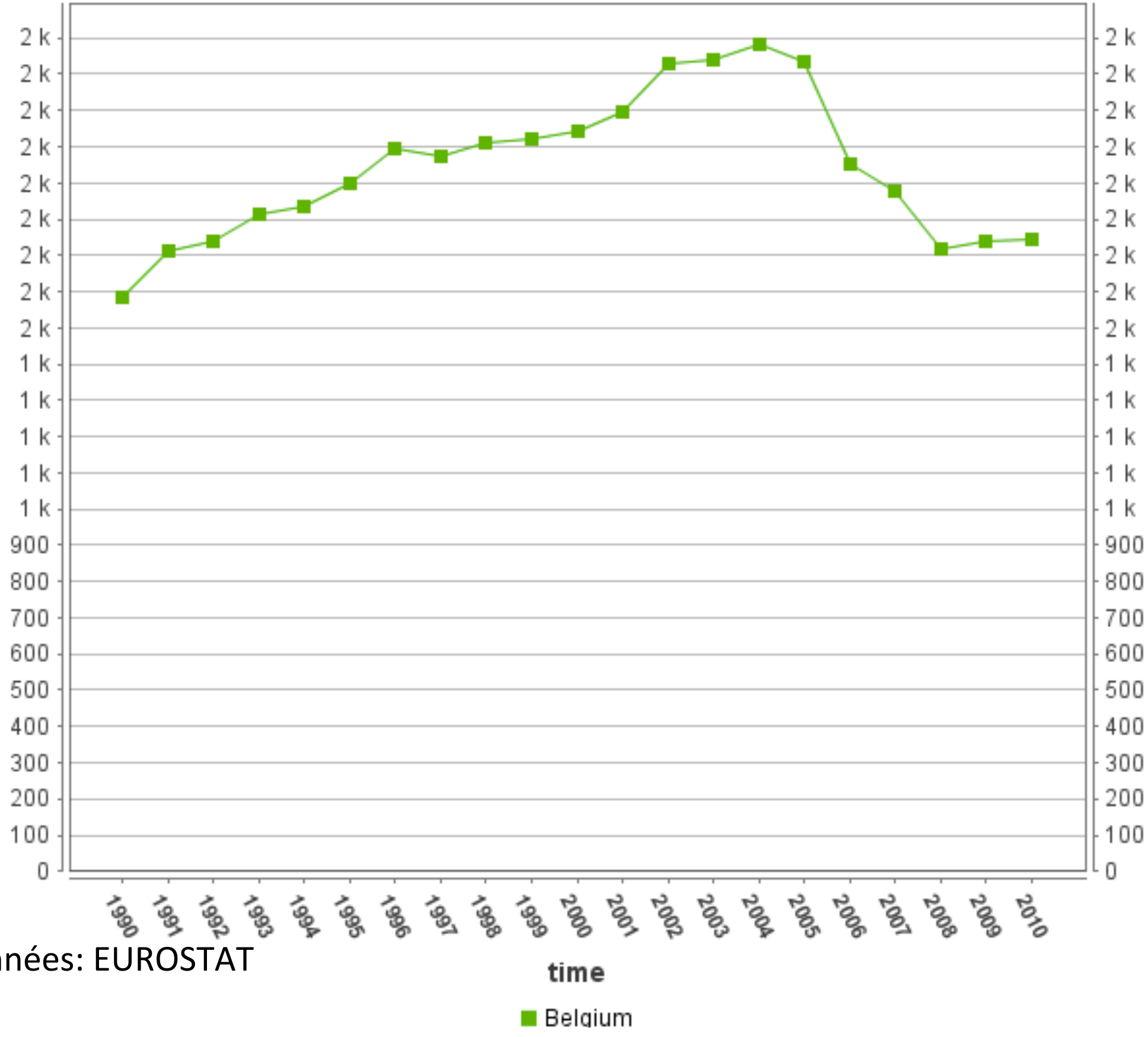




\section{Europe 2020}

- Des objectifs ambitieux

- Mais réalistes?

- La réalisation des objectifs E2020 nécessite

- Une participation très large

- Une politique ambitieuse

- Une stratégie de mise en œuvre claire 
MARCHÉ LIBÉRALISÉ DE L'ÉLECTRICITÉ 


\section{L'électricité, un bien particulier}

- Dans un réseau électrique, à tout moment, la production doit être égale à la consommation (aux pertes près)

- Baisse de la tension, black-out

- Sur un réseau électrique, les flux électriques obéissent aux lois de Kirchoff 


\section{Les métiers de l'électricité}

- Dans le domaine de l'électricité, on distingue 4 'métiers'

- Production

- Commercialisation

- Transport (réseau HT)

- Distribution (réseaux MT et BT) 


\section{Economies d'échelle}

- Le coût des infrastructures du transport (et de distribution) correspond principalement à un coût fixe:

- Investissement

- maintenance

- Le coût dépend peu de l'intensité d'utilisation, de sorte qu'une utilisation plus intense des réseaux permet d'amortir leurs coûts sur un plus grand nombre d'unités.

- Si le coût marginal est constant, le coût moyen du réseau diminue avec la quantité de services offerts (économies d'échelle)

- Les économies d'échelle peuvent être telles que la duplication de l'infrastructure par un concurrent n'est pas économiquement possible

- monopole de fait 


\section{Le monopole intégré}

- Pendant longtemps, le marché de l'électricité s'est organisé sous la forme d'un monopole intégré

- Une entreprise responsable des 4 métiers, à l'exception éventuellement de la distribution

- Organisation monopolistique justifiée par les économies d'échelle

- Régulation des activités du monopole

- Tarifaire

- Incorporation des gains de productivité et progrès technique

- Coordination aisée des différentes activités 


\section{Remise en cause du modèle monopolistique}

- Capacité limitée du régulateur à contrôler le monopole

- Inefficacité dans l'allocation des ressources

- Introduction de mécanismes concurrentiels pour accroître l'efficacité

- Abaissement des tailles minimales efficaces

- Volonté de l'Europe de créer un marché Européen intégré 


\section{Le marché libéralisé}

- La libéralisation est organisée autours de 4 principes

1. Séparation verticale

2. Accès ouvert

3. Ouverture à la concurrence

4. Régulation résiduelle 


\section{Séparation verticale}

- Séparation des différents métiers de l'électricité

- Séparation fonctionnelle (plusieurs business units)

- Séparation juridique

- Séparation patrimoniale

- Chacune des activités a un prix

- Les relations entre les différentes entités s'organisent sur une base contractuelle et commerciale et la coordination des activités évolue vers un système basé sur les prix. 


\section{Accès ouvert}

- Accès de la production et de la commercialisation aux infrastructures de transport et de distribution

- Principe de l'accès transparent et nondiscriminatoire

- Tarification de type 'timbre poste' 


\section{Ouverture à la concurrence}

- La production et la commercialisation d'électricités sont ouvertes à la concurrence

- La concurrence se justifie par

- L'absence de rendements d'échelle

- La baisse des tailles efficaces minimales

- Le jeu de la concurrence devrait 'en principe' faire baisser le prix du KWh d'électricité

- Les producteurs ont accès aux réseau HT et BT moyennant le paiement d'une redevance d'accès 


\section{Ouverture à la concurrence}

- En Wallonie depuis 2007, les consommateurs ont le choix de leur fournisseur d'électricité

- Fournisseur par défaut pour assurer la continuité de l'offre

- Choix de plusieurs formules tarifaires (fixe vs variable, mix énergétique, prix au Kwh, durée du contrat)

- Comparateur de prix (test achat, CWAPE) 


\section{Régulation résiduelle}

- Le transport et la distribution conserve une structure monopolistique

- Justification: économies d'échelle

- Les activités de transport et de distribution sont régulées

- Transport: régulateur fédéral (CREG)

- Distribution: projet de régionalisation

- Obligations de service public imposées par les régions 


\section{Les composantes de la facture}

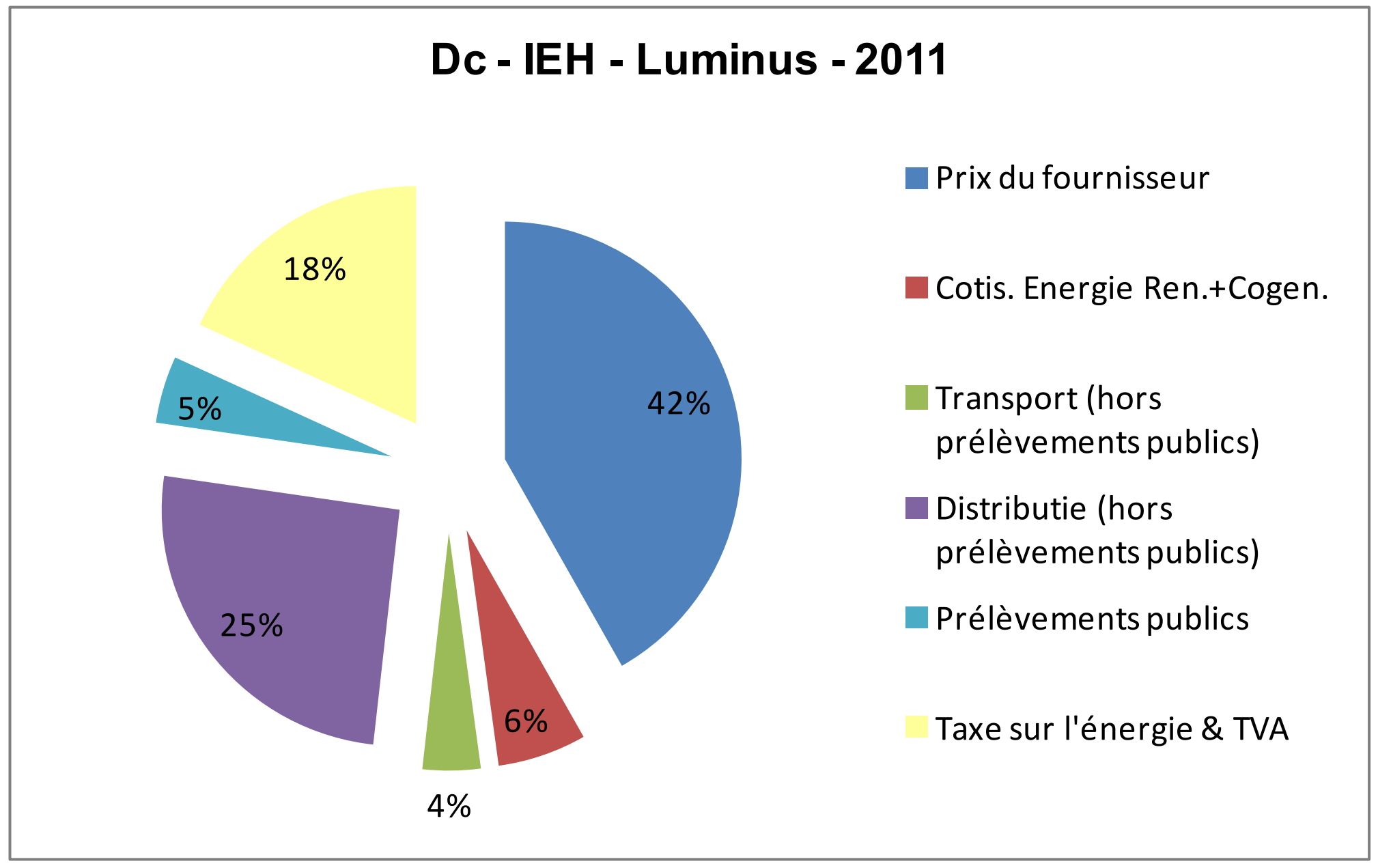

Source: Creg (2011)

Simulation réalisée sur base d'une consommation annuelle de 3500 kW 
- Les acteurs

\section{En Belgique}

- Production

- Electrabel

- SPE (Luminus)

- Autres (Nuon, Lampiris,...)

- Importations (7\% de la consommation en 2005)

- Transport: ELIA

- Distribution: Intercommunales

- Régulateurs: CREG (National), Cwape (Wallonie), VREG (Flandre), Breugel (Bruxelles) 


\section{Part de marché, Wallonie 2009}

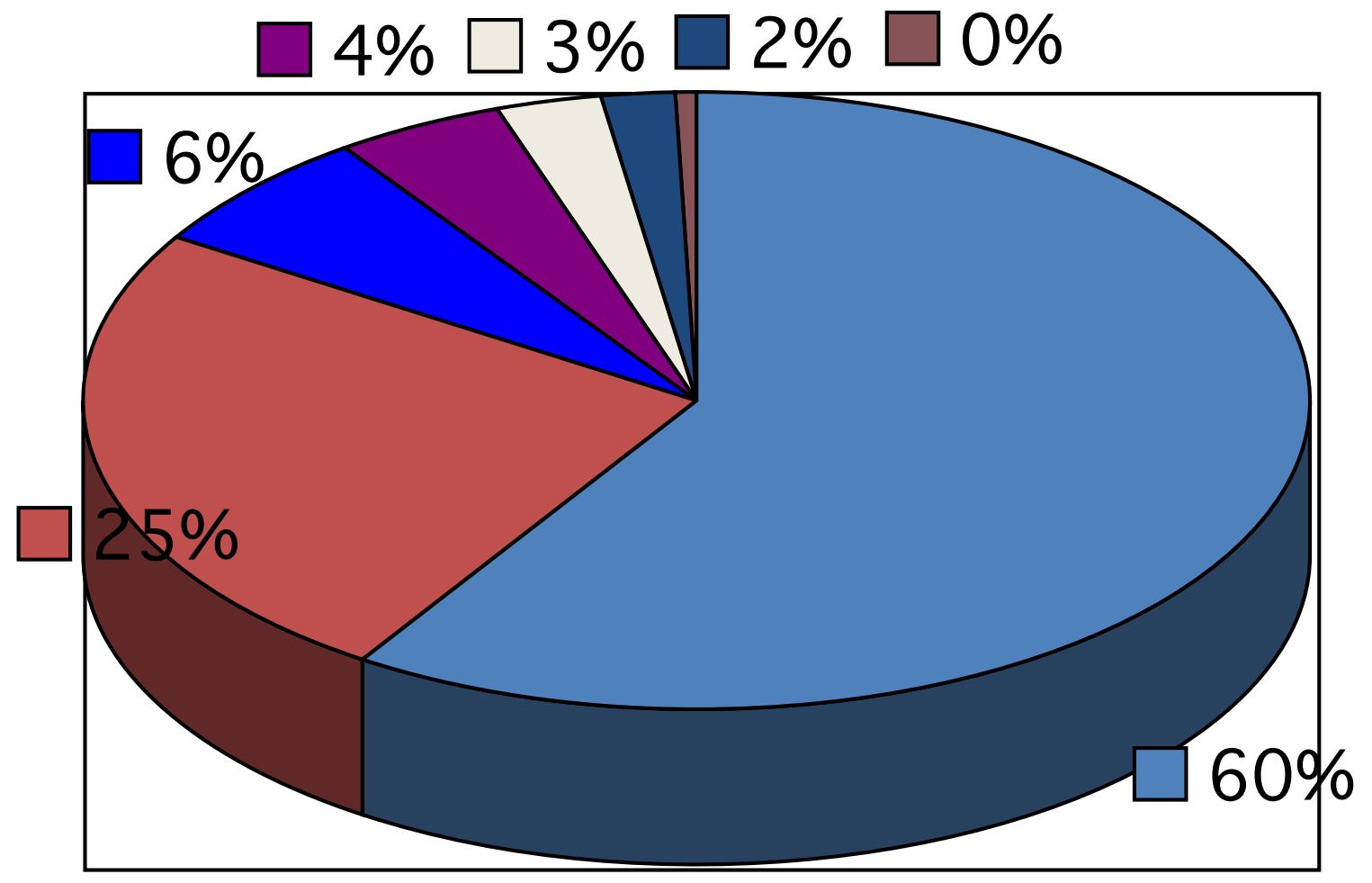

$\square$ Electrabel

$\square$ SPE

$\square$ Essent BE

$\square$ Lampiris

$\square$ Nuon BE

$\square$ GRD

$\square$ Autres

Source: CWAPE 


\section{En Belgique}

- Pour les consommateurs, pas de baisse substantielle de la facture d'énergie depuis la libéralisation

- Prix des combustibles fossiles

- Concurrence limitée

- Entreprise dominante

- Hausse importante des tarifs de distribution

- Augmentation des taxes

- Augmentation de la part des énergies vertes fortement subsidiées 


\section{Les défis}

- Une régulation plus efficace

- Une concurrence plus intense

- Investissement dans de nouvelles capacités

- de production

- d'interconnexion

- Développement et intégration sur les réseaux des énergies renouvelables

- Développement des réseaux 


\section{LES ÉNERGIES RENOUVELABLES}




\section{Définition}

- Prélevée sur des flux naturels et non pas sur des stocks qui ne se renouvellent pas

- Principales sources d'énergies renouvelables:

- Eolien

- Solaire

- Hydraulique

- Géothermie

- Biomasse

- Marée

$-\ldots$ 


\section{Production d'énergie}

Graphique 18 Part des différentes formes d'énergie dans la production brute d'électricité, scénario de référence en \% sur le graphique de gauche, en TWh sur le graphique de droite
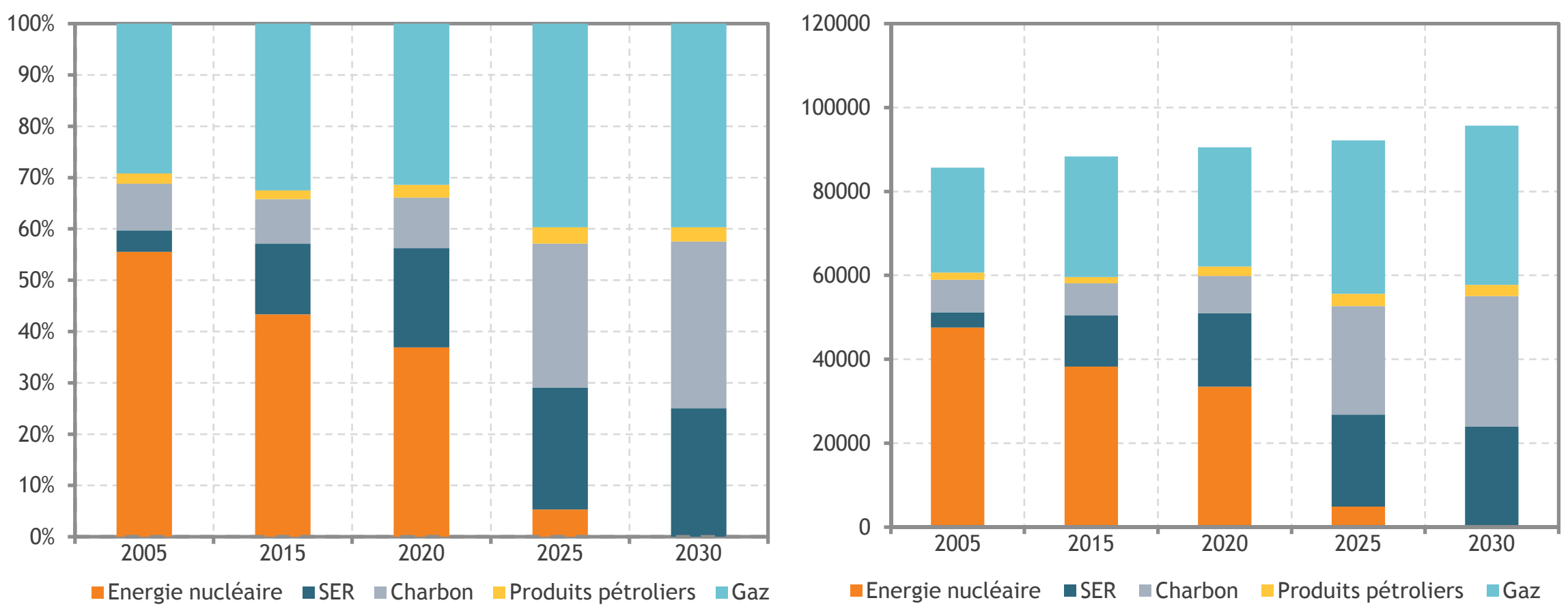

Sources : Eurostat, PRIMES

Données: Bureau du plan Perspectives énergétiques pour la

Belgique à l'horizon 2030 


\section{Energies renouvelables}

Graphique 19 Production brute d'électricité à partir de sources d'énergie renouvelables, scénario de référence en \% sur le graphique de gauche, en TWh sur le graphique de droite
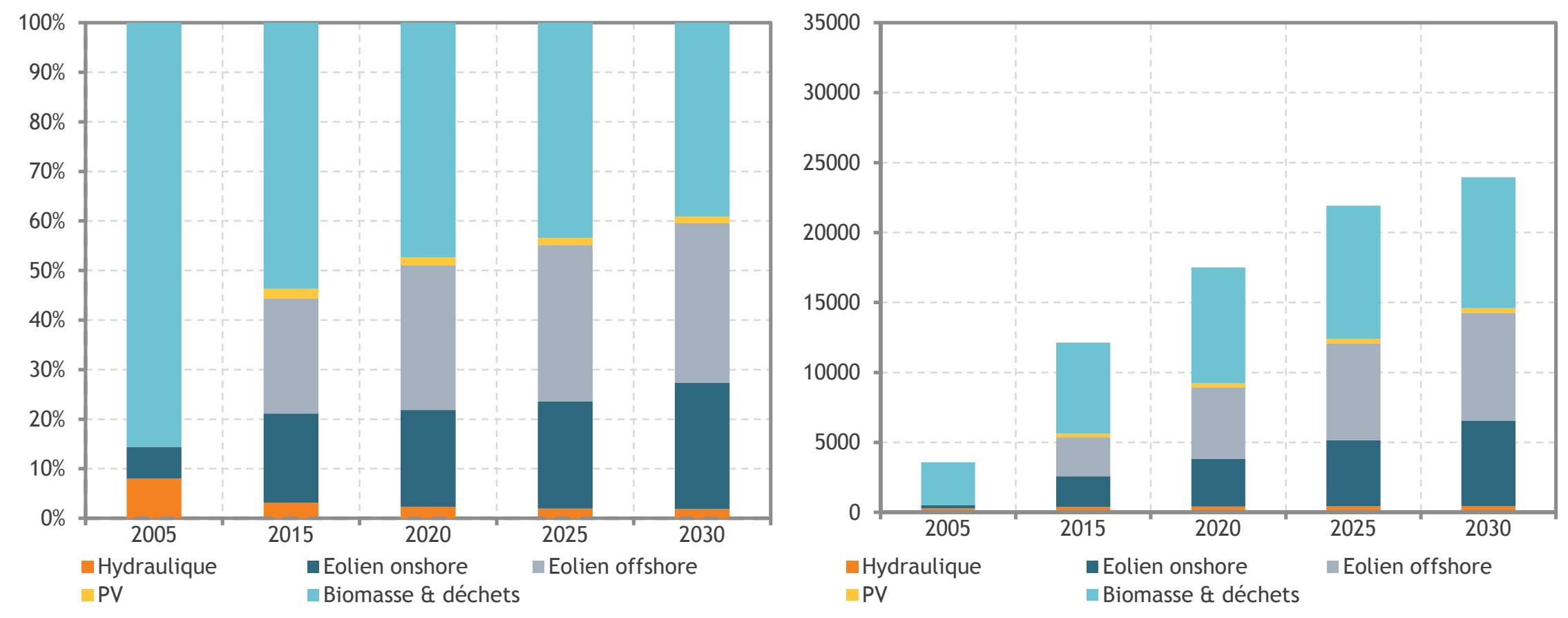

Sources : Eurostat, PRIMES.

Données: Bureau du plan Perspectives énergétiques pour la Belgique à l'horizon 2030 


\section{Wallonie, capacité en 2010}

\begin{tabular}{lcc} 
Sites de production & Nombre de sites & Puissance (kW) \\
\hline Solaire PV & 21276 & 85908 \\
Hydraulique & 83 & 110204 \\
Éolien & 61 & 431308 \\
Biomasse & 8 & 93727 \\
Cogénération biomasse & 49 & 158430 \\
Cogénération fossile & 60 & 167861 \\
\hline Total & $\mathbf{2 1 5 3 7}$ & $\mathbf{1 0 4 7 4 3 8}$
\end{tabular}

Tableau 6 : Sites de production d'électricité verte fin 2010 


\section{Wallonie, 2010}

Puissance installée : 1047 MWe

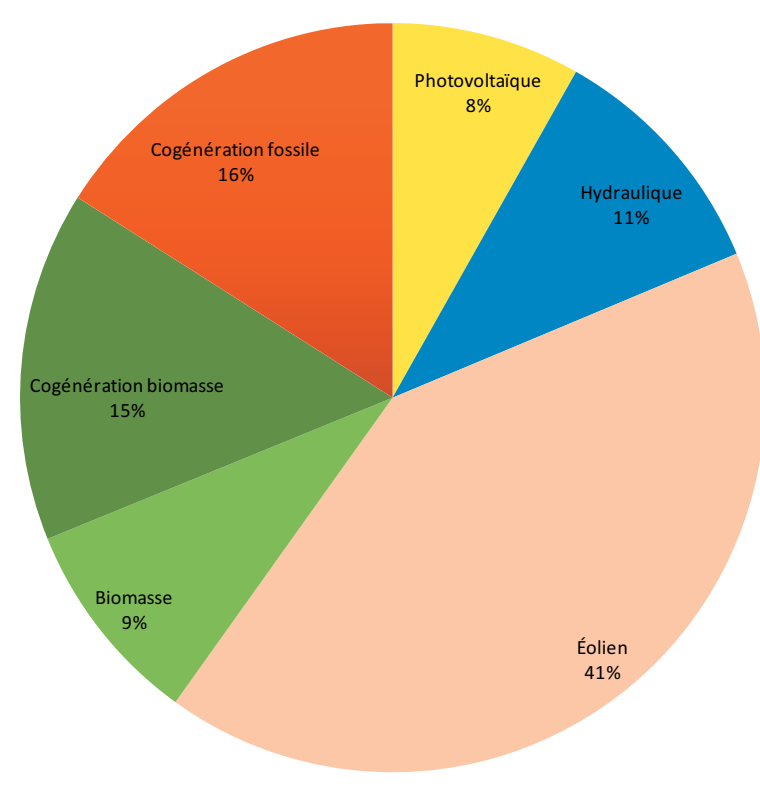

3491 GWh produits

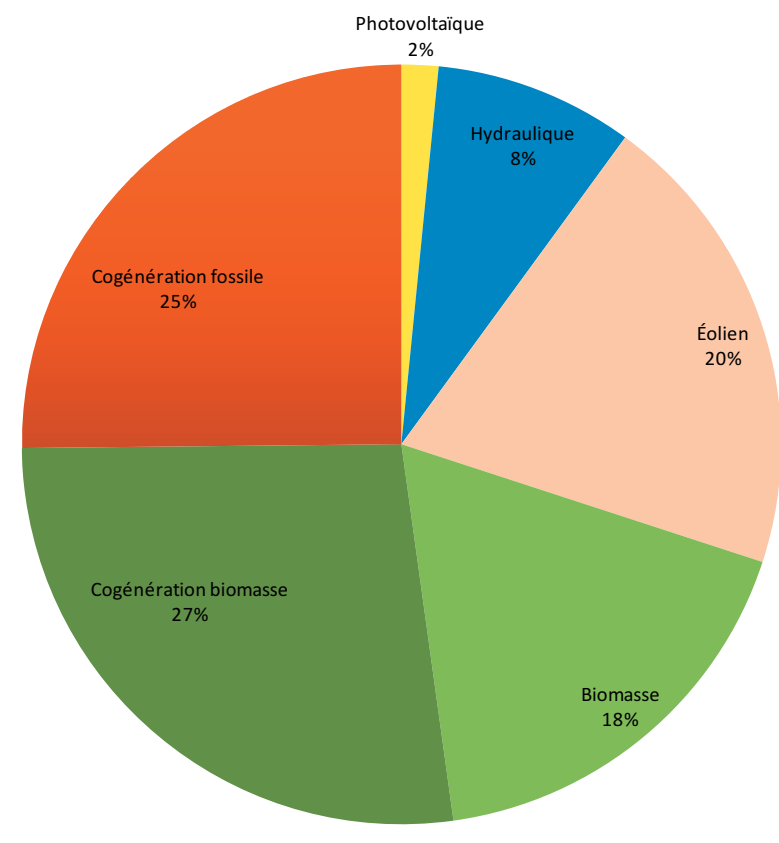




\section{Des énergies chères}

\begin{tabular}{|c|c|c|c|c|c|}
\hline & Nucléaire & $\begin{array}{l}\text { Eolien } \\
\text { Off-shore }\end{array}$ & $\begin{array}{l}\text { Eolien } \\
\text { On-shore }\end{array}$ & TGV & Charbon \\
\hline $\begin{array}{l}\text { Puissance } \\
\text { (MW) }\end{array}$ & 1400 & 5 & 2 & 480 & 750 \\
\hline $\begin{array}{l}\text { Durée de vie } \\
\text { (années) }\end{array}$ & 60 & 20 & 25 & 30 & 40 \\
\hline $\begin{array}{l}\text { Taux } \\
\text { d'émission } \\
\text { CO2 }\end{array}$ & 0 & 0 & 0 & 56 & 93 \\
\hline $\begin{array}{l}\text { Coût marginal } \\
\text { de long terme } \\
€ / \mathrm{MWh}\end{array}$ & 50,89 & 148,90 & 83,89 & 60,52 & 64,03 \\
\hline
\end{tabular}

Source: Hansen \& Percebois (2010) 


\section{L'énergie éolienne}

- Coût de production de $85 € / M W h$

- $38 \%$ plus chère que le gaz

- L'énergie éolienne est

- Intermittente

- Imprévisible

- Le prix perçu par les producteurs est relativement faible

- Les éoliennes ne sont pas économiquement rentable en l'absence d'un mécanisme de soutien 


\section{Soutien aux énergies vertes}

- En Wallonie, mise en place d'un système de certificats verts pour soutenir la production d'énergies renouvelables

- Les producteurs se voient accorder un certain nombre de CV pour chaque MW produit à partir d'énergies renouvelables

- Les CV ont une valeur comprise entre $65 €$ et $100 €$ 


\section{Les CV}

- Les fournisseurs d'énergie doivent fournir au régulateur des CV correspondant à pourcentage prédéfini de leurs livraisons

- En cas de défaut, une amende de $100 €$ par CV manquant est appliquée

- Il y a un marché pour les CV

- La RW garantit un prix minimum de $65 €$

- Prix en 2011: $83 €$ 


\section{Octroi des CV en Wallonie}

\begin{tabular}{|l|c|c|c|}
\hline $\begin{array}{l}\text { Filières } \\
\text { (et puissance totale d'installation) }\end{array}$ & $\begin{array}{c}\text { Taux } \\
\text { d'octroi } \\
\text { (CV/MWh) }\end{array}$ & $\begin{array}{c}\text { Niveau de soutien } \\
\text { minimum garanti } \\
\text { (EUR/MWh) }\end{array}$ & $\begin{array}{c}\text { Niveau de soutien } \\
\text { maximum théorique } \\
\text { (EUR/MWh) }\end{array}$ \\
\hline Cogénération fossile $(\leq 20 \mathrm{MW})$ & 0,1 à 0,4 & 6,5 à 25 & 10 à 40 \\
\hline Biomasse $(\leq 20 \mathrm{MW})$ & 0,1 à 1 & 6,5 à 65 & 10 à 100 \\
\hline Hydraulique $(\leq 20 \mathrm{MW})$ & 1 & 65 & 100 \\
\hline Éolien & 1 & 65 & 100 \\
\hline Cogénération biomasse $(\leq 5 \mathrm{MW})$ & 0,1 à 2 & 6,5 à 130 & 600 à 700 \\
\hline Photovoltaïque $(\leq 10 \mathrm{kWc})$ & 6 à 7 & 390 à 455 & 170 à 600 \\
\hline Photovoltaïque $(10-250 \mathrm{kWc})$ & 1,2 à 6 & 160 à 390 & 150 à 170 \\
\hline Photovoltaïque $(>250 \mathrm{kWc})$ & 1 à 1,2 & 150 à 160 & \\
\hline
\end{tabular}

Tableau 1 : Niveau de soutien pour différentes filières de production

Cwape, 2010 


\section{Quotas de CV}

\begin{tabular}{|l|l|}
\hline 2003 & $3 \%$ \\
\hline 2004 & $4 \%$ \\
\hline 2005 & $5 \%$ \\
\hline 2006 & $6 \%$ \\
\hline 2007 & $7 \%$ \\
\hline 2008 & $8 \%$ \\
\hline 2009 & $9 \%$ \\
\hline 2010 & $10-11.75 \%$ \\
\hline 2011 & $13.5 \%$ \\
\hline 2012 & $15.75 \%$ \\
\hline
\end{tabular}




\section{Utilisation des éoliennes}

\section{Table 2}

Average capacity factors over 2003-2007.

\begin{tabular}{lrrrrrrrrr}
\hline Area & EU15 & DE & ES & DK & IT & UK & FR & PT & NL \\
\hline Capacity (GW) & 56.3 & 22.2 & 14.1 & 3.1 & 2.7 & 2.5 & 2.4 & 2.2 & 1.7 \\
Energy (TWh) & 97.7 & 39.5 & 28.8 & 6.1 & 4.2 & 5.3 & 4.2 & 3.8 & 3.5 \\
Load share (\%) & 3.2 & 6.2 & 8.5 & 15.6 & 1.3 & 1.3 & 0.7 & 7.0 & 3.3 \\
Capacity factor (\%) & 20.8 & 18.3 & 24.8 & 22.8 & 19.1 & 26.1 & 22.3 & 22.7 & 21.5 \\
& & & & & & & & & \\
Area & AT & GR & IR & SE & BE & PL & FI & CA & US \\
\hline Capacity (GW) & 1.0 & 0.9 & 0.8 & 0.7 & 0.3 & 0.3 & 0.1 & 2.4 & 16.6 \\
Energy (TWh) & 2.0 & 1.9 & 1.9 & 1.2 & 0.5 & 0.5 & 0.2 & 4.4 & 32.1 \\
Load share (\%) & 3.1 & 2.9 & 16.2 & 0.8 & 0.5 & 0.3 & 0.2 & 1.9 & 0.7 \\
Capacity factor (\%) & 20.1 & 29.3 & 29.3 & 21.7 & 20.0 & 25.9 & 21.8 & 22.4 & 25.7 \\
\hline & & & & & & & & &
\end{tabular}

Source: Boccard (2009) Energy Policy 


\section{Intermittence et imprévisibilité}

- L'énergie éolienne est intermittente et imprévisible

- En Belgique, le facteur d'utilisation est de l'énergie éolienne on-shore est de $20 \%$

- Pour palier aux problèmes d'intermittence et d'imprévisibilité

- Marché spot

- Capacités additionnelles de production 


\section{Production}

- Tableau de production avec $\mathbf{N}$ générateurs intermittents

- $\quad C F=20 \%$

- Générateurs non-corrélés

\begin{tabular}{|lll|}
\hline & $\mathrm{N}=3$ & $\mathrm{~N}=10$ \\
\hline $0 \mathrm{MWH}$ & $51,2 \%$ & $11 \%$ \\
\hline $1 \mathrm{MWh}$ & $38,4 \%$ & $27 \%$ \\
\hline $2 \mathrm{MWh}$ & $9,6 \%$ & $7 \%$ \\
\hline $3 \mathrm{MWh}$ ou + & $0,8 \%$ & $55 \%$ \\
\hline
\end{tabular}




\section{Production}

- La multiplication de générateurs non-corrélés permet de pallier en partie les problèmes d'intermittence des générateurs

- L'introduction de l'énergie éolienne ne peut se concevoir qu'en conjonction avec d'autres types de générateurs

- Pour avoir des générateurs non-corrélés: investir dans l'interconnexion des réseaux 


\section{Perspectives}

- Couplage des énergies éoliennes avec d'autres générateurs pour résoudre les problèmes d'intermittence

- Nécessiter de penser en terme de mix énergétique

- L'évaluation économique et environnementale de l'énergie éolienne doit se faire sur un base d'un mix énergétique 


\section{Perspectives}

- Couplage de l'énergie éolienne avec

- Dispositifs de type 'smart grid'

- Dispositifs de stockage

- Mécanisme de réserve (non-consommation)

- Augmentation des capacités d'interconnexion pour diversifier les sources d'approvisionnement 


\section{CONCLUSIONS}




\section{Pour conclure}

- Objectifs Europe 2020 ambitieux

- L'intégration des énergies renouvelables dans les réseaux électriques nécessite de repenser ceux-ci

- Investissement

- Progrès technique

- La réalisation des objectifs ne pourra se faire sans un soutien public efficace 
Je vous remercie pour votre attention!

\author{
Axel Gautier \\ Université de Liège \\ agautier@ulg.ac.be
}

